\title{
Soziale Ungleichheit und Risiken der Erwerbsminderung
}

\author{
Christine Hagen/Ralf K. Himmelreicher \\ Daniel Kemptner/Thomas Lampert
}

\begin{abstract}
Sozialepidemiologische Forschung und Gesundheitsberichterstattung liefern inzwischen zahlreiche empirische Hinweise darauf, dass zwischen der sozialen und gesundheitlichen Lage der Bevölkerung ein enger Zusammenhang besteht. Die Analyse der Zugänge in Erwerbsminderungsrenten leistet hier zweierlei: Sie bestätigt die bisherigen Befunde auf einer breiten und verlässlichen Datenbasis und zeigt die Effekte sozialer Ungleichheit im Hinblick auf die Inanspruchnahme einer wohlfahrtsstaatlichen Leistung. Gerade die unterschiedliche Betroffenheit nach Qualifikation unterstreicht die Notwendigkeit von Bildung für ein langes und gesundes Arbeitsleben.
\end{abstract}

\section{Einleitung}

Viele chronische Krankheiten und Beschwerden treten bei Personen, die in Bezug auf Qualifikation, Berufsstatus und Einkommen als benachteiligt angesehen werden können, vermehrt auf und nehmen zudem oftmals einen ungünstigeren Verlauf (Mielck 2000, 2005; Lampert et al. 2005; Richter/Hurrelmann 2009). In Deutschland basieren die meisten Untersuchungen zum Zusammenhang zwischen der sozialen und gesundheitlichen Lage auf Daten bevölkerungsrepräsentativer Gesundheitssurveys (Kurth et al. 2009). Zunehmende Bedeutung kommt den Routinedaten der Sozialversicherungsträger zu (Himmelreicher/Stegmann 2008; Brockmann et al. 2009; Hagen et al. 2010). Eine vielversprechende Datenbasis für die sozialepidemiologische Forschung und Gesundheitsberichterstattung, die bislang kaum genutzt wird, stellen die Scientific Use Files des Forschungsdatenzentrums der Rentenversicherung (FDZ-RV) dar. Im Folgenden werden diese herangezogen, um soziale Unterschiede beim Zugang in Erwerbsminderungsrenten (EM-Renten) zu untersuchen.

Bei einer Berentung wegen Erwerbsminderung kommt chronischen Krankheiten, wie z.B. Herz-Kreislauf-Leiden, Bandscheibenvorfällen und schweren Depressionen große Bedeutung zu. Wer aufgrund solcher Krankheiten eine Erwerbstätigkeit nicht oder nur noch eingeschränkt verrichten kann, hat - sofern die Voraussetzungen erfüllt sind - Anspruch auf eine Erwerbsminderungsren- te. Das frühzeitige Ausscheiden aus dem Erwerbsleben durch Frühberentung stellt für die Betroffenen zumeist ein gravierendes krankheitsbezogenes Ereignis dar. Der Frühberentung geht zum einen häufig eine längere Krankheitsgeschichte voraus, die zu langfristigen Einschränkungen der Gesundheit und Leistungsfähigkeit führt. Zum anderen ist der Bezug einer EM-Rente mit finanziellen Einbußen verbunden (Dragano et al. 2008).

Im Mittelpunkt des vorliegenden Beitrags steht die Frage, inwiefern sich soziale Ungleichheit in der Inanspruchnahme von EM-Renten ausdrückt. Der Fokus liegt dabei auf Herz-Kreislauf-, Muskel-Skelettund psychischen Erkrankungen, die prozentual zu den häufigsten Diagnosegruppen der Frühberentung zählen. Soziale Unterschiede im Rentenzugang werden in erster Linie an der höchsten schulischen und beruflichen Qualifikation von Frauen und Männern in den alten und neuen Bundesländern festgemacht. Dabei wird davon ausgegangen, dass der Qualifikation hohe Bedeutung für das Risiko einer krankheitsbedingten Frühberentung zukommt. Sie hat nicht nur Einfluss auf die beruflichen Chancen und den zu erreichenden Lebensstandard, sondern auch auf die körperlichen und psychischen Arbeitsbelastungen. Erwerbstätige mit niedrigem Qualifikationsniveau üben häufiger Berufe aus, die mit chronischen Fehlbelastungen und psychischem Stress verbunden sind (Dragano 2007; Marmot/Wilkonson 1999; Robert/ House 2000). Hochqualifizierte können eher selbstbestimmt arbeiten und sie haben geringere Dequalifizierungs-, Arbeitslosigkeits- und gesundheitliche Risiken (Radl 2007). In diesem Zusammenhang ist auch die geschlechtsspezifische Segregation des
Arbeitsmarktes und ihre Auswirkung auf die Inanspruchnahme von EM-Renten zu berücksichtigen. Frauen und Männer sind nach wie vor unterschiedlichen Arbeitsumgebungen und Formen von Anforderungen und Belastungen in der Arbeitswelt ausgesetzt. Während die Arbeitsbedingungen von Männern zumeist höhere physische Gesundheitsrisiken aufweisen (Beermann et. al. 2008; Kuhn 2008; Lademann/Kolip 2005; Koppelin/Müller

Christine Hagen, Dr., ist wissenschaftliche
Mitarbeiterin im Fachgebiet Gesundheits-
berichterstattung am Robert Koch-Institut
(RKI) in Berlin. Arbeitsschwerpunkte: Soziale
und gesundheitliche Ungleichheit, Gender-
forschung, Gesundheitsberichterstattung.
e-mail: c.hagen@rki.de
Ralf K. Himmelreicher, PD Dr., ist Referent
im Forschungsdatenzentrum der Rentenver-
sicherung (FDZ-RV) und Lehrbeauftragter
an der Freien Universität Berlin. Arbeits-
schwerpunkte: Einkommen und Vermögen
im Lebenslauf, Verteilungsfragen, empirische
Wirtschafts- und Sozialforschung.
e-mail: ralf.himmelreicher@drv-bund.de
Daniel Kemptner, Dipl.-Volkswirt, ist
Doktorand am Graduate Center, Deutsches
Institut für Wirtschaftsforschung (DIW)
Berlin. Arbeitsschwerpunkte: Empirische
Arbeitsmarkt- und Gesundheitsökonomik,
angewandte Ökonometrie.
e-mail: dkemptner@diw.de
Thomas Lampert, Dr., ist stellvertretender
Leiter des Fachgebiets Gesundheitsbericht-
erstattung am RKI. Arbeitsschwerpunkte:
Soziale und gesundheitliche Ungleichheit,
Gesundheit im Lebenslauf, Gesundheits-
berichterstattung.
e-mail: t.lampert@rki.de


2004), wird in zahlreichen Studien auf erhöhte berufsbedingte psychische Belastungen von Frauen verwiesen (Beermann et al. 2008; BiBB/BAuA 2006; Europäische Agentur für Sicherheit und Gesundheitsschutz am Arbeitsplatz 2006). Neben den unterschiedlichen Arbeitsbelastungen spielen sicherlich auch noch andere Faktoren eine Rolle: Männer zeigen häufiger eine riskante Lebensweise (Brandes 2003; Sticher 2010), neigen darüber hinaus eher zur Bagatellisierung von Symptomen und nehmen das Gesundheitswesen seltener oder später in Anspruch (RKI 2006). Darüber hinaus stellt sich die Frage, ob die weitgehende Angleichung der Morbidität und Mortalität zwischen den alten und neuen Bundesländern (RKI 2009) sich auch im Berentungsgeschehen ausdrückt oder ob frühere Unterversorgungsphasen (Schmidtke 1997) sowie anhaltende schlechtere Bedingungen des Arbeitsmarktes (Frommert/Himmelreicher 2010) zu regionalen Ungleichheiten hinsichtlich der krankheitsbedingten Frühberentung führen.

Im Folgenden werden zunächst die gesetzlichen Grundlagen der Erwerbsminderungsrente beschrieben (Abschnitt 2). Abschnitt 3 stellt die zugrunde liegende Datenquelle und die Methode dar. In Abschnitt 4 präsentieren wir die Befunde und diskutieren sie im abschließenden Abschnitt 5.

\section{Institutionelle Rahmen- bedingungen}

Im Jahr 1957 wurden die Renten wegen Berufsunfähigkeit (BU) und wegen Erwerbsunfähigkeit (EU) einheitlich für Arbeiter und Angestellte durch das Rentenreformgesetz (RRG 1957) modifiziert. Zum 1.1.2001 trat das Gesetz zur Reform der Renten wegen verminderter Erwerbsfähigkeit (EM-ReformG) in Kraft: Damit wurden die bisherigen Renten wegen Berufs- und Erwerbsunfähigkeit für Neufälle abgelöst (\$ 43 SGB VI). Nicht zuletzt aufgrund der Einführung des EM-ReformG haben die Zugänge in Erwerbsminderungsrente vor allem bei Männern bis 2008 stetig abgenommen (Brussig/Wojtkowski 2006, S. 5), ${ }^{1}$ da die Anspruchsvoraussetzungen verschärft wurden (Dannenberg et al. 2010, S. 287).
Die krankheitsbedingte EM-Rente versichert insbesondere abhängig Beschäftigte gegen das Risiko einer dauerhaften Arbeitsunfähigkeit und die damit verbundenen materiellen Risiken. Entscheidendes Kriterium dafür, ob Versicherte eine EM-Rente erhalten, ist, inwieweit die berufliche Leistungsfähigkeit einer Person auf dem allgemeinen Arbeitsmarkt durch Krankheit beeinträchtigt ist: Bei einem Leistungsvermögen von sechs und mehr Stunden pro Tag besteht kein Anspruch auf eine EM-Rente; sind Versicherte in der Lage von drei bis unter sechs Stunden täglich zu arbeiten, erhalten sie eine Rente wegen teilweiser Erwerbsminderung; ist es für Versicherte nicht möglich, mindestens drei Stunden täglich erwerbstätig zu sein, erhalten sie eine volle Erwerbsminderungsrente. $^{2}$

Das Verfahren zur Feststellung einer EM-Rente wird auf Antrag des Versicherten vom zuständigen Rentenversicherungsträger eröffnet. Grundlage ist das Gesetz zur Rente wegen Erwerbsminderung ( $\$ 43$ SGB VI). Wesentliche Voraussetzungen zum Bezug einer EM-Rente, neben dem bereits genannten reduzierten Arbeitsvermögen, sind:

- Regelaltersgrenze für die Altersrente ist noch nicht erreicht,

- Erwerbsfähigkeit kann durch Rehabilitationsmaßnahmen nicht mehr hergestellt werden,

- die Wartezeit ist erfüllt, d. h. die Antragstellenden sind seit mindestens fünf Jahren in der gesetzlichen Rentenversicherung (gRV) versichert.

- Ferner müssen innerhalb der letzten fünf Jahre vor Erwerbsminderung drei Jahre lang Pflichtbeiträge abgeführt worden sein. ${ }^{3}$

Der medizinische Teil des Verfahrens zur Feststellung einer EM-Rente beruht auf ärztlichen Befunden und sozialmedizinischen Diagnosen bestellter Gutachter. Nach Dragano et al. (2008, S. 110) „(..) ist mit einiger Sicherheit davon auszugehen, dass der Umstand einer bewilligten Erwerbsminderungsrente ein valider Indikator für eine zugrunde liegende schwerwiegende Erkrankung ist." Hinzuweisen ist jedoch auf eine gewisse Selektivität des EM-Rentenbezugs: zum einen, weil nicht die gesamte Erwerbsbevölkerung versichert ist. Zum anderen, weil einige Versicherte trotz Krankheit keinen Antrag auf EM-Rente stellen und deshalb zum Beispiel häufiger arbeitsunfähig werden, oder weil sie sich krankheitsbedingt eine weniger belastende berufliche Tätigkeit, u.U. in einem Teilzeitbeschäftigungsverhältnis, gesucht haben. Zudem kann eine vorangegangene erfolgreiche Rehabilitationsmaßnahme die Arbeitsfähigkeit wiederhergestellt haben. Ferner können Beschäftigte auf anderen Wegen ihre Erwerbsbiografien beenden, z. B. im Rahmen von Arbeitslosigkeit oder anderen Altersrentenarten.

Die EM-Renten sind eine bedeutende Leistung der gesetzlichen Rentenversicherung. Knapp jede fünfte Rente, die die gRV neu ausbezahlt, ist eine EM-Rente; damit wurden seit 2005 jährlich rund 160.000 EM-Renten erstmals ausbezahlt (DRV 2009a). Die durchschnittlichen Rentenzahlbeträge lagen hier 2008 bei etwa $600 €$ (DRV 2009b) und stellen bei etwa jedem zweiten EM-Rentner die einzige persönliche Einkommensquelle dar (Albrecht et al. 2007). Weil Niveausenkungen weder durch eine Verlängerung der Lebensarbeitszeit noch durch verstärkte private Altersvorsorge kompensiert werden können (Köhler-Rama et al. 2010), sind EM-Rentner besonders vom Paradigmenwechsel in der Alterssicherung - in Richtung verstärkter Eigenvorsorge - betroffen (Viebrok et al. 2004). Speziell durch die Einführung von Abschlägen bei Inanspruchnahme einer Erwerbsminderungsrente vor Vollendung des 63. Lebensjahres, trotz gleichzeitiger Verlängerung der Zurechnungszeit vom Alter 55 Jahre auf das Alter 60 Jahre, sind die durchschnittlichen Rentenzahlbeträge von vollen EM-Renten im Zeitraum von 2000 bis 2008 nominal von $738 €$ auf $647 €$ gesunken (DRV 2009a). Wegen dieser enormen Einbußen fordert die aktuelle Bundesregierung in ihrem Koalitionsvertrag, „(...) dass auch erwerbsgeminderte Menschen angemessen sozial abgesichert

1 Im Jahr 2000 wurden in Deutschland insgesamt 214.082 EM-Rentenzugänge gezählt, im Jahr 2008 waren es lediglich 162.839, was einem Rückgang von 24 \% entspricht; bei Männern ist im vergleichbaren Zeitraum ein Rückgang von knapp $35 \%$ zu verzeichnen (vgl. DRV 2009a, S. 78, 79)

2 Der Rentenartfaktor, der multiplikativ in die Rentenberechnung eingeht, beträgt bei vollen EMRenten 1,0 und bei teilweisen EM-Renten 0,5.

3 Möglicherweise verkürzte Wartezeiten sind in $\S 53$ SGB VI (Vorzeitige Wartezeiterfüllung) geregelt. 
sind (...)" (Koalitionsvertrag 2009). ${ }^{4}$ Allerdings ist eine zusätzliche Absicherung gegen das Risiko von Einkommensarmut im Erwerbsminderungsfall bei privaten Versicherungen insbesondere für besonders gefährdete und häufig gering qualifizierte Berufsgruppen entweder nicht oder nur zu hohen Kosten zu bekommen.

\section{2 \\ Datenbasis und Methode}

Empirische Grundlage der Analysen zu sozialen Unterschieden beim Zugang in Erwerbsminderungsrenten sind prozessproduzierte Daten der Deutschen Rentenversicherung, die Informationen über den in der gesetzlichen Rentenversicherung versicherten Personenkreis enthalten. ${ }^{5}$ Diese werden vom Forschungsdatenzentrum der Rentenversicherung (FDZ-RV ${ }^{6}$ ) aufbereitet und stehen wissenschaftlichen Forschungseinrichtungen für nicht kommerzielle Forschungsvorhaben kostenfrei zur Verfügung. Folgende Datensätze, die die jeweilige Grundgesamtheit repräsentieren, wurden im Rahmen des „Kontrollierten Fernrechnens" genutzt: Zur Analyse der Zugänge in eine Erwerbsminderungsrente wird der Themendatenfile „Erwerbsminderung und Diagnosen (RTZN08XVSTEM)“ des Jahres 2008 verwendet, begrenzt auf solche EM-Rentenzugänge, die erstmals eine Erwerbsminderungsrente beziehen. Prozessproduzierte Daten der Rentenversicherung ermöglichen Analysen u.a. im Bereich chronischer Erkrankungen, die qua bewilligter Erwerbsminderungsrente operationalisiert werden. Hierbei beruht der medizinische Teil des Verfahrens zur Feststellung einer EM-Rente aufärztlichen Befunden und sozialmedizinischen Diagnosen bestellter Gutachter. Damit ist der Zugang in diese Rente ein valider Indikator für eine zugrunde liegende schwerwiegende Erkrankung. In dem Datensatz sind Informationen zu Krankheiten enthalten und nach ICD-10-GM Version in Haupt- und Nebendiagnosen verschlüsselt. Aus den differenzierten ICD-10-GM Diagnosen werden Diagnosegrundgruppen erstellt, in denen ähnliche Diagnosen zusammengefasst sind (vgl. DRV 2009b, S. 342). In den Analysen werden die drei häufig vorkommenden Diagnosegruppen HerzKreislauf-Erkrankungen, Muskel-Skelett-

Tabelle 1: Ausbildung und Qualifikationsniveau bei Versicherten und Zugängen in Erwerbsminderungsrente (2008) - Anteile und Fallzahlen* -

\begin{tabular}{|c|c|c|c|}
\hline Qualifikationsniveau & Ausbildung & aktiv Versicherte & $\begin{array}{l}\text { Zugänge in } \\
\text { EM-Rente }\end{array}$ \\
\hline niedriges & $\begin{array}{l}\text { Haupt-/Realschulabschluss } \\
\text { ohne Berufsausbildung }\end{array}$ & $\begin{array}{c}8,50 \% \\
(2.219 .041)\end{array}$ & $\begin{array}{l}14,78 \% \\
(18.797)\end{array}$ \\
\hline \multirow[t]{3}{*}{ mittleres } & $\begin{array}{l}\text { Haupt-/Realschulabschluss } \\
\text { mit Berufsausbildung }\end{array}$ & $\begin{array}{c}47,10 \% \\
(12.303 .283)\end{array}$ & $\begin{array}{l}46,06 \% \\
(58.583)\end{array}$ \\
\hline & $\begin{array}{l}\text { Abitur ohne } \\
\text { Berufsausbildung }\end{array}$ & $\begin{array}{c}0,73 \% \\
(190.594)\end{array}$ & $\begin{array}{l}0,33 \% \\
(426)\end{array}$ \\
\hline & $\begin{array}{l}\text { Abitur mit } \\
\text { Berufsausbildung }\end{array}$ & $\begin{array}{c}3,84 \% \\
(1.003 .574)\end{array}$ & $\begin{array}{l}1,48 \% \\
(1.880) \\
\end{array}$ \\
\hline \multirow[t]{2}{*}{ hohes } & Fachhochschule & $\begin{array}{c}3,23 \% \\
(843.475)\end{array}$ & $\begin{array}{l}1,11 \% \\
(1.410)\end{array}$ \\
\hline & Universität/Hochschule & $\begin{array}{c}5,0 \% \\
(1.305 .001) \\
\end{array}$ & $\begin{array}{l}1,19 \% \\
(1.520)\end{array}$ \\
\hline Total & mit validen Bildungsangaben & $\begin{array}{c}68,4 \% \\
(17.864 .968)\end{array}$ & $\begin{array}{l}64,95 \% \\
(82.616)\end{array}$ \\
\hline \multirow[t]{2}{*}{ keine Beobachtung } & Unbekannt & $\begin{array}{c}21,21 \% \\
(5.539 .413)\end{array}$ & $\begin{array}{l}23,64 \% \\
(30.066)\end{array}$ \\
\hline & Keine Angabe & $\begin{array}{c}10,39 \% \\
(2.714 .990)\end{array}$ & $\begin{array}{l}11,41 \% \\
(14.517)\end{array}$ \\
\hline \multicolumn{2}{|l|}{ Insgesamt } & $\begin{array}{c}100 \% \\
(26.119 .371)\end{array}$ & $\begin{array}{c}100 \% \\
(127.199)\end{array}$ \\
\hline \multicolumn{4}{|c|}{$\begin{array}{l}\text { * Höchste schulische und berufliche Qualifikation, inländische 30-59-jährige } \\
\text { aktiv Versicherte. }\end{array}$} \\
\hline \multicolumn{4}{|c|}{ Quelle: FDZ-RV - RTZN08XVSTEM, AKVS08XVSBB; Berechnungen der Autoren. } \\
\hline
\end{tabular}

Erkrankungen und psychische Erkrankungen berücksichtigt. Um EM-Rentenrisiken berechnen zu können, werden als Kontrollgruppe die „Aktiv Versicherten“7 ebenfalls des Jahres 2008 (AKVS08XVSBB) genutzt, begrenzt auf solche, die im Berichtsjahr keine Rente und keine Beschäftigungszeiten nach dem Altersteilzeitgesetz bezogen haben. ${ }^{8}$ In beiden Datensätzen wurde die Untersuchungspopulation auf in Deutschland lebende versicherte Personen (Inlandskonzept) im Alter von 30 bis einschließlich 59 Jahren eingegrenzt. Ferner werden ausschließlich erstmals eine EM-Rente beziehende Personen betrachtet; ausgeschlossen werden Personen mit Vorerkrankungen, wie z.B. Behinderte, Rehabilitanden und Pflegepersonen. ${ }^{9}$ Als Merkmal sozialer Ungleichheit fungieren in beiden Datensätzen die vergleichbar kodierten Merkmale zur Ausbildung, die in drei Qualifikationsniveaus gruppiert wurden (Tabelle 1). ${ }^{10}$

Grundsätzlich ist zum Merkmal Ausbildung anzumerken, dass es nicht rechts-

4 Es soll geprüft werden, wie Erwerbsminderungsrisiken in der staatlich geförderten Vorsorge kostenneutral verbessert werden können. Kostenneutral bedeutet dabei, dass EM-Risiken ohne staatliche oder betriebliche Förderung von den Beschäftigten zu finanzieren sind, und zwar im Rahmen der bereits geförderten Altersvorsorge, à la "Erwerbsminderungs-Riester". Ein solches Produkt würde die Altersvorsorge verringern, weil bei einem gegeben Beitrag ein weiteres Risiko zu versichern wäre, oder Arbeitnehmern würden höhere Kosten entstehen. erheblich ist; d.h. es beeinflusst nicht die Rentenhöhe, weshalb es weniger valide ist als rentenrelevante Merkmale. Zudem liegt in einigen Fällen keine Meldung vor (vgl. FN 10). Zudem weisen Personen ohne valide Bildungsangaben u.a. plausible Alters-Lohn-Profile in etwa von niedrig

5 Zum versicherten Personenkreis zählen Personen, die gegen ein Arbeitsentgelt beschäftigt sind (Kreikebohm-Kuzynski 2011, S. 383ff.). Von den Daten nicht repräsentiert sind ausschließlich geringfügig Beschäftigte, Beamte und das Gros der Selbstständigen.

6 Zum Datenangebot und den Zugangswegen siehe www.fdz-rv.de.

7 Diese Eingrenzung schließt alle Versicherten ein die im Berichtsjahr eine Beitrags- oder eine Anrechnungszeit haben. Beitragszeiten sind Zeiten, für die überwiegend Pflichtbeiträge an die Rentenversicherung abgeführt wurden, wg. Beschäftigung oder wg. Zeiten der Kindererziehung. Anrechnungszeiten sind bei Vorliegen der Voraussetzungen Zeiten, in denen eine versicherte Tätigkeit z. B. wegen Arbeitsunfähigkeit, Arbeitslosigkeit, Schwanger- und Mutterschaft nicht erfolgt.

8 Zur Beschreibung der beiden Datensätze siehe die jeweiligen Codepläne unter www.fdz-rv.de.

9 Diese Personen können über die Variable TTSC1 identifiziert werden.

10 Zur Zuordnung der Variable Ausbildung in den Datensätzen des FDZ-RV in Bildungsniveaus siehe u. a Himmelreicher et al. (2009). Das Merkmal Ausbildung stammt aus der Meldung der Arbeitgeber zur Sozialversicherung (DEÜV) und wird seit dem Jahr 2000 in den RV-Daten gespeichert und seit dieser Zeit mit jeder neuen Meldung überschrieben. Insbesondere bei langjährig (seit 2000) Arbeitslosen, Minijobbern, passiv Versicherten (Haufrauen und -männern), sowie bei inzwischen Selbstständigen und Beamten liegt häufig keine Meldung vor. 
Qualifizierten auf, die erheblich unter denen von Mittel- oder Hochqualifizierten liegen (Fachinger/Himmelreicher 2007). Insofern dürften fehlende Bildungsangaben bei eher arbeitsmarktferneren, zum Teil auch gesundheitlich beeinträchtigten Personen auftreten. Fehlende Werte bei der Bildungsvariable gehen jedoch von Jahr zu Jahr zurück: Zum einen, weil vom Einführungstermin des Merkmals zunehmend weniger Fälle betroffen sind, und zum anderen, weil Maßnahmen der Qualitätssicherung im statistischen Berichtswesen der Rentenversicherung greifen. oder versicherten Arbeitseinkommen, die - wegen Geringfügigkeits- und Beitragsbemessungsgrenze - in der Rentenversicherung links- und rechtszensiert sind, reflektiert das Bildungsmerkmal sowohl etwa humankapitaltheoretisch abgeleitete Einkommenserzielungspotenziale (Fachinger/Himmelreicher 2007) als auch bildungsgeprägtes Gesundheitsverhalten. Es repräsentiert somit ein adäquates Merkmal zur Untersuchung von Gesundheit und sozialer Ungleichheit.

Sowohl bei den aktiv Versicherten als auch bei Personen, die 2008 erstmalig EMRente beziehen, dominiert das mittlere Qualifikationsniveau (Tabelle 1). Bei den EM-Rentenzugängen ist jedoch im Vergleich zu den aktiv Versicherten der Anteil mit niedrigem Qualifikationsniveau höher und der Anteil mit hohem Qualifikationsniveau geringer. Zudem ist festzustellen, dass fehlende Bildungsangaben bei den $\mathrm{Zu}$ gängen in EM-Rente häufiger vorkommen als bei den Versicherten. Dies ist darauf zurückzuführen, dass der Altersdurchschnitt bei den EM-Rentenzugängen über jenem der aktiv Versicherten liegt und für jüngeIm Unterschied zu z. B. Entgeltpunkten

re Versicherte häufiger - seit der Einführung des Ausbildungsmerkmals im Jahr 2000 - eine valide Bildungsangabe vom Arbeitgeber vorliegt als für ältere. Trotz der Ausfälle aufgrund fehlender Bildungsangaben stellen 82.616 EM-Rentenzugänge und 17.864.968 inländische 30- bis 59-jährige aktiv Versicherte mit Angaben zum Qualifikationsniveau eine Datenbasis dar, mit der soziale Unterschiede beim EM-Rentenzugangsgeschehen analysiert werden können. ${ }^{11}$

\section{1 \\ Empirische Befunde}

Tabelle 2 weist die relativen Zugänge in Erwerbsminderungsrenten im Verhältnis zu den entsprechenden aktiv Versicherten aus. Diese Verhältnisse werden im Folgenden als relative Erwerbsminderungsrisiken (EM-Risiken pro 1.000 Versicherte) interpretiert. Es zeigt sich, dass ostdeutsche Männer das höchste EM-Risiko aufweisen, gefolgt von ostdeutschen Frauen und westdeutschen Männern. Bei westdeutschen Frauen sind die geringsten EM-Risiken zu verzeichnen. Differenziert man die globalen EM-Risiken nach häufig vorkommenden Diagnosegruppen, so zeigt sich, dass psychische Beschwerden der häufigste Grund für den Bezug einer EM-Rente sind, wobei insbesondere westdeutsche Frauen hier vergleichsweise hohe EM-Risiken aufweisen. Sogenannte „Verschleißerkrankungen“ der Muskeln, des Skeletts und des Bindegewebes erweisen sich als der zweithäufigste Grund für eine EM-Rente. Bei ostdeutschen Män-

\section{Tabelle 2: Zugänge in Erwerbsminderungsrente nach Region, Geschlecht und Diagnosegruppen (2008) - Anteil pro 1.000 aktiv Versicherte - Fallzahlen in Klammern*}

\begin{tabular}{|c|c|c|c|c|}
\hline & \multicolumn{2}{|c|}{ Westdeutschland } & \multicolumn{2}{|c|}{ Ostdeutschland } \\
\hline & Männer & Frauen & Männer & Frauen \\
\hline \multirow{2}{*}{$\begin{array}{l}\text { Zugang in die } \\
\text { Erwerbsminderungsrente } \\
\text { (insgesamt) }\end{array}$} & 4,71 & 4,60 & 6,43 & 5,28 \\
\hline & $(51.293)$ & $(47.212)$ & $(15.797)$ & $(12.858)$ \\
\hline $\begin{array}{l}\text { davon wegen psychischer } \\
\text { Erkrankungen }\end{array}$ & $\begin{array}{c}1,50 \\
(16.335)\end{array}$ & $\begin{array}{c}2,00 \\
(20.527)\end{array}$ & $\begin{array}{c}1,60 \\
(3.931)\end{array}$ & $\begin{array}{c}1,85 \\
(4.505)\end{array}$ \\
\hline $\begin{array}{l}\text { davon wegen Herz-Kreislauf- } \\
\text { Erkrankungen }\end{array}$ & $\begin{array}{c}0,62 \\
(6.752)\end{array}$ & $\begin{array}{c}0,25 \\
(2.566)\end{array}$ & $\begin{array}{c}1,02 \\
(2.506)\end{array}$ & $\begin{array}{l}0,39 \\
(949)\end{array}$ \\
\hline $\begin{array}{l}\text { davon wegen Muskel-Skelett- } \\
\text { Erkrankungen }\end{array}$ & $\begin{array}{c}0,70 \\
(7.622)\end{array}$ & $\begin{array}{c}0,68 \\
(6.979)\end{array}$ & $\begin{array}{c}1,14 \\
(2.801)\end{array}$ & $\begin{array}{c}0,89 \\
(2.167)\end{array}$ \\
\hline \multicolumn{5}{|c|}{$\begin{array}{l}\text { * Grundgesamtheit sind inländische 30-59-jährige Versicherte. Die ausgewiesenen } \\
\text { Fallzahlen beziehen sich auf die Zugänge in Erwerbsminderungsrente insgesamt bzw. } \\
\text { nach Diagnosegruppen. }\end{array}$} \\
\hline \multicolumn{5}{|c|}{ Quelle: FDZ-RV - RTZN08XVSTEM, AKVS08XVSBB; Berechnungen der Autoren. } \\
\hline
\end{tabular}

nern sind diesbezüglich die höchsten Risiken zu konstatieren: Annähernd jeder neunhundertste ostdeutsche versicherte Mann bezieht wegen einer chronischen muskuloskelettalen Erkrankung eine EMRente. Bei Herz-Kreislauf-Erkrankungen haben Männer im Vergleich zu Frauen ein höheres Risiko, wobei hier ebenfalls der hohe Anteil von ostdeutschen Männern auffällt.

Abbildung 1 zeigt die Entwicklung der EM-Risiken mit zunehmendem Alter der weiblichen und männlichen Versicherten in den alten und neuen Bundesländern. Während diese Risiken bei 30- bis 45-Jährigen vergleichsweise gering sind, steigt die Risikoquote bei den Älteren leicht exponentiell - weitgehend unabhängig von der Region - bis zum 57. Lebensjahr. Allerdings mit einer Ausnahme: westdeutsche Männer im Alter von 50 Jahren. Ihr erhöhtes EM-Risiko ist auf die rentenrechtliche Besonderheit zurückzuführen, dass Bergleute, wenn sie im Bergbau vermindert berufsfähig sind, gewisse rentenrechtliche Voraussetzungen wie knappschaftliche Pflichtbeitragszeiten erfüllen und das 50 . Lebensjahr vollendet haben, eine Rente beziehen können ( $\$ 45$ SGB VI). Das nach dem 57. Lebensjahr abflachende EMRenten-Risiko ist darauf zurückzuführen, dass manche Versicherte versuchen, ihre Berentung in Richtung Altersrente (ab 60 Jahre) ohne Gesundheitsprüfung zu verschieben.

Am Ende des fünften Lebensjahrzehnts beziehen etwa 18 von 1.000 Männern in Ostdeutschland eine EM-Rente. In Westdeutschland sind es etwa 16 von 1.000 Männern. Die EM-Risiken von ost- und westdeutschen Frauen liegen ungefähr auf einem Niveau und ab einem Alter von 55 Jahren deutlich unter dem der - insbesondere ostdeutschen - Männer.

Abbildung 2 weist EM-Risiken differenziert nach Qualifikationsniveaus und Geschlecht der Versicherten in der Erwerbsphase aus. Es zeigt sich sehr deutlich, dass in jeder Altersgruppe Geringqualifizierte höhere EM-Risiken haben als höher Qualifizierte. Ferner liegen die EM-Risiken bei Frauen mit niedrigem und mittlerem Qualifikationsniveau unter denen der vergleichbaren Männer. Lediglich bei Versicherten

11 Bei der Interpretation der Befunde ist zu berücksichtigen, dass sich mit steigendem Stichprobenumfang die Varianz der Schätzwerte der logistischen Regressionen verringert. 
mit hohem Qualifikationsniveau sind bei Männern im Alter zwischen 40 bis Mitte 50 Jahren niedrigere EM-Risiken zu konstatieren als bei Frauen; allerdings erfolgt eine Annäherung der EM-Risiken von hochqualifizierten Männern und Frauen bis zum Alter von 59 Jahren. Der knappschaftliche Alterseffekt bei 50-Jährigen zeigt sich insbesondere bei niedrig qualifizierten Bergmännern. Diese Befunde weisen darauf hin, dass die höchste schulische und berufliche Qualifikation mit dem Risiko einer Erwerbsminderung in Zusammenhang steht: (Fach-)Hochschulabsolventen weisen ein wesentlich geringeres Risiko auf, wegen chronischen Krankheiten frühzeitig ihre Erwerbstätigkeit unterbrechen zu müssen, als geringer Qualifizierte. Die EM-Risiken niedrig qualifizierter Männer und Frauen sind dabei etwa sieben- bzw. drei- bis viermal so hoch wie die der hochqualifizierten Vergleichsgruppen.

Die Abbildungen 1 und 2 gemeinsam interpretiert legen nahe, dass es sich bei westdeutschen Hochqualifizierten um einen Personenkreis handelt, der geringe EM-Risiken aufweist. Um den Einfluss mehrerer Variablen - im Hinblick auf die Wahrscheinlichkeit, in einem bestimmten Jahr eine EM-Rente zu beziehen - schätzen zu können, werden logistische Regressionsmodelle benutzt. Die ausgewiesenen Odds Ratios geben an, um welchen Faktor die EM-Risiken im Verhältnis zur definierten Referenzkategorie (Westdeutsche und/ oder Hochqualifizierte) erhöht sind. Diese EM-Risikoverhältnisse werden zur sprachlichen Vereinfachung in den Tabellen 3 und 4 EM-Risikoquoten genannt.

Sämtliche in Tabelle 3 ausgewiesenen Schätzergebnisse sind auf dem $5 \%$-Niveau signifikant. Im Vergleich zu den westdeutschen Hochqualifizierten (Referenzkategorie) haben geringer qualifizierte Gruppen höhere EM-Renten-Risiken.

Dies gilt für beide Geschlechter, wobei jedoch das Qualifikationsniveau bei Männern das EM-Risiko wesentlich stärker als bei Frauen beeinflusst. Deutlich wird zudem, dass die EM-Renten-Risiken in den neuen Bundesländern höher sind als in den alten, auch hier fallen die Unterschiede bei den Männern größer aus. Die EM-RentenRisiken von in Ostdeutschland lebenden Männern mit niedrigem Qualifikationsniveau sind rund zehnmal, die der ostdeutschen niedrigqualifizierten Frauen rund viermal größer als die von westdeutschen Hochqualifizierten.

\section{Abb. 1: Erwerbsminderungsrenten-Risiken nach Geschlecht* und Region 2008}

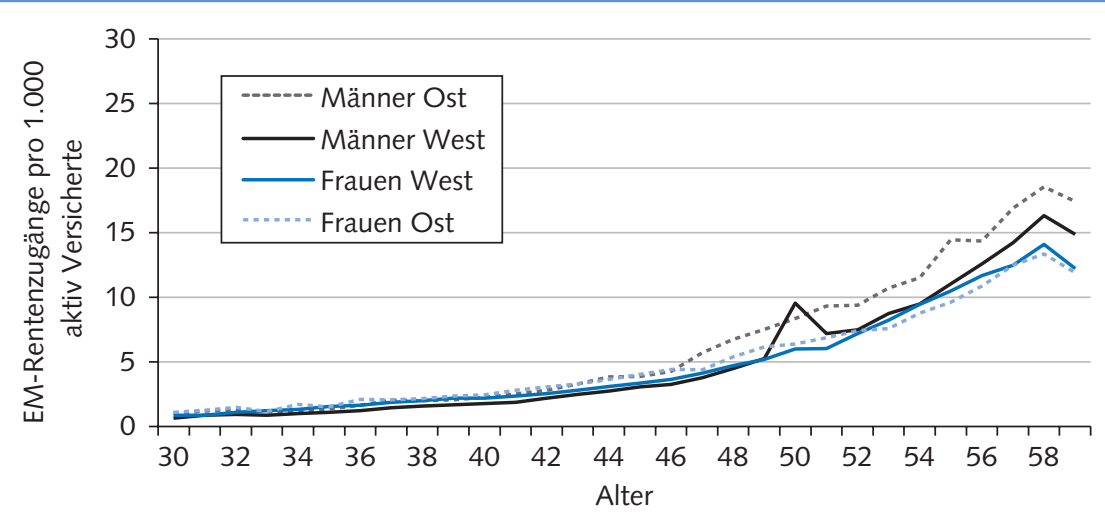

*inländische 30-59-jährige Versicherte.

Quelle: FDZ-RV - RTZN08XVSTEM, AKVS08XVSBB Berechnungen der Autoren.

WSI MITTEILUNGEN

Abb. 2: Erwerbsminderungsrenten-Risiken in Deutschland 2008*

- differenziert nach Qualifikationsniveau -

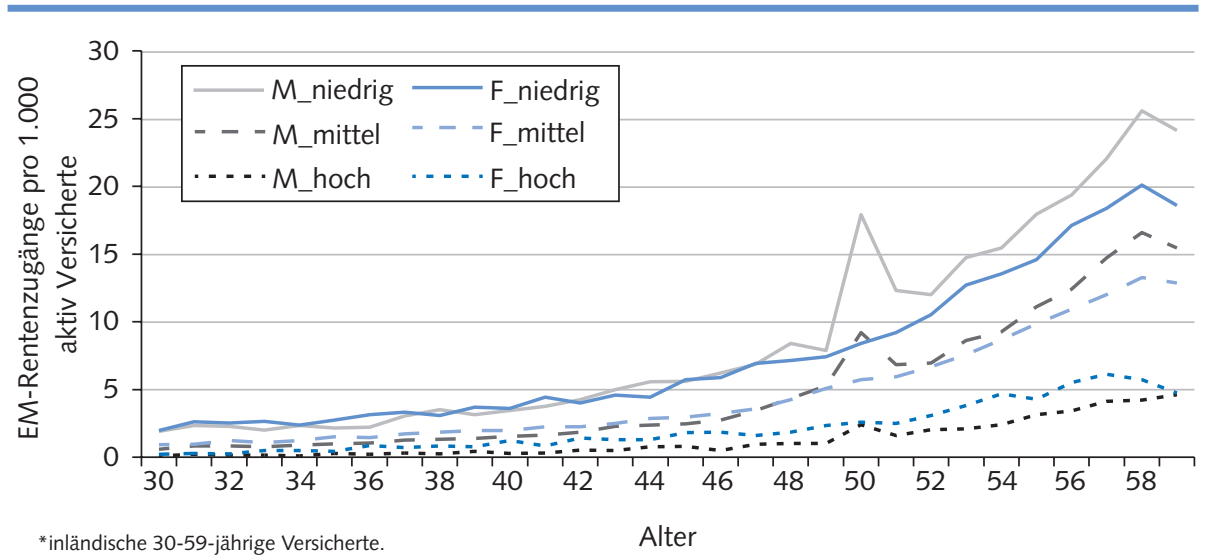

Quelle: FDZ-RV - RTZN08XVSTEM, AKVS08XVSBB;

WSI MITTELUNGEN

Tabelle 3: Risikoquoten (Odds-Ratios) zur Erwerbsminderungsrente nach Qualifikation und Region (2008) - logistische Regression+ ${ }^{+}$

\begin{tabular}{|c|c|c|}
\hline Erklärende Variablen & Männer & Frauen \\
\hline $\begin{array}{l}\text { Niedriges Qualifikationsniveau - } \\
\text { Ostdeutschland }\end{array}$ & $\begin{array}{l}10,44^{*} \\
(0,4681)\end{array}$ & $\begin{array}{c}4,37^{*} \\
(0,2132)\end{array}$ \\
\hline $\begin{array}{l}\text { Mittleres Qualifikationsniveau - } \\
\text { Ostdeutschland }\end{array}$ & $\begin{array}{c}5,49 * \\
(0,1761)\end{array}$ & $\begin{array}{l}2,32^{*} \\
(0,081)\end{array}$ \\
\hline $\begin{array}{l}\text { Hohes Qualifikationsniveau - } \\
\text { Ostdeutschland }\end{array}$ & $\begin{array}{c}1,43^{*} \\
(0,0834)\end{array}$ & $\begin{array}{c}1,00 \\
(0,056)\end{array}$ \\
\hline $\begin{array}{l}\text { Niedriges Qualifikationsniveau - } \\
\text { Westdeutschland }\end{array}$ & $\begin{array}{c}7,52^{*} \\
(0,2411)\end{array}$ & $\begin{array}{c}3,45^{*} \\
(0,1195)\end{array}$ \\
\hline $\begin{array}{l}\text { Mittleres Qualifikationsniveau - } \\
\text { Westdeutschland }\end{array}$ & $\begin{array}{c}3,94^{*} \\
(0,1214)\end{array}$ & $\begin{array}{c}2,11^{*} \\
(0,0708)\end{array}$ \\
\hline $\begin{array}{l}\text { Hohes Qualifikationsniveau - } \\
\text { Westdeutschland }\end{array}$ & \multicolumn{2}{|c|}{ Referenzkategorie } \\
\hline Pseudo- $R^{2}$ & 0,0753 & 0,0497 \\
\hline Beobachtungen & 9.504 .952 & 8.339 .746 \\
\hline
\end{tabular}

Im Folgenden werden Erwerbsminderungsrisiken differenziert nach Qualifi- kationsniveau, Geschlecht und Region für die drei häufig vorkommenden Diagno- 
segruppen Herz-Kreislauf-Erkrankungen, Muskel-Skelett-Erkrankungen und psychische Erkrankungen dargestellt (Tabelle 4).

Bezüglich EM-Rentenzugängen wegen Herz-Kreislauf-Erkrankungen sowie wegen Muskel-Skelett-Erkrankungen fallen vor allem die stark erhöhten Risiken bei Männern mit niedriger und mittlerer Qualifikation im Vergleich zu den hochqualifizierten Männern auf. Bei Frauen sind bei diesen Gruppen ebenfalls deutlich erhöhte Risiken festzustellen. Darüber hinaus ist bei beiden Erkrankungen das EM-RentenRisiko bei Männern wie Frauen in den neuen Bundesländern höher als in den alten Bundesländern.

Bei EM-Rentenzugängen aufgrund von psychischen Erkrankungen sind wie bei Herz-Kreislauf- sowie Muskel-Skelett-Erkrankungen ebenfalls Qualifikationsunterschiede festzustellen, allerdings auf deutlich niedrigerem Niveau. Männer mit niedriger Qualifikation haben ein etwa 7-fach erhöhtes Risiko im Verhältnis zu hochqualifizierten Männern; bei Frauen beträgt das entsprechende Verhältnis ungefähr 3 zu 1. Deutlich geringer als bei den beiden anderen Diagnosegruppen fallen damit die Qualifikations- wie auch die Ost-West-Unterschiede aus. Bei Frauen ist das mit psychischen Erkrankungen verbundene EM-Renten-Risiko in Ostdeutschland sogar etwas niedriger als in Westdeutschland. Die vergleichsweise geringeren sozialen Unterschiede hinsichtlich der psychischen Erkrankungen lassen sich möglicherweise darauf zurückführen, dass insbesondere psychische Belastungen prinzipiell in jedem (Arbeits-)Leben an jedem Arbeitsplatz auftreten können.

\section{Diskussion}

Untersuchungen in Deutschland zum Zusammenhang zwischen sozialer und gesundheitlicher Lage basieren überwiegend auf Daten von bevölkerungsrepräsentativen Befragungen mit vergleichsweise geringem Stichprobenumfang. Mehrdimensionale Analysen verschiedener chronischer Krankheiten mit regionalem, geschlechtsspezifischem und vor allem erwerbsbiografischem Bezug scheitern dabei in der Regel an der Fallzahlenproblematik. Im Unterschied zu solchen Forschungsdesigns wurden für die vorliegende Studie prozessproduzierte Daten der gesetzlichen Rentenversicherung benutzt, genauer: die Grundgesamtheit aller Rentenzugänge in eine Erwerbsminderungsrente sowie als Kontrollgruppe die aktiv Versicherten im Jahr 2008. Die Analysen für die zugrunde liegende Untersuchungspopulation - in Deutschland lebende versicherte Personen im Alter von 30 bis einschließlich 59 Jahren im Jahr 2008 - zeigen, dass die EM-Renten-Risiken ungleich verteilt sind: Sowohl die Qualifikation als auch das Geschlecht und der Wohnort haben einen signifikanten Einfluss auf das Risiko, eine Erwerbminderungsrente zu beziehen. Diese Befunde sind von gesellschafts- wie finanzpolitischer Bedeutung, weil chronische Erkrankungen bei gesundheitsbedingten Frühberentungen (EM-Rente) eine besondere Rolle spielen: Nahezu jeder fünfte Rentenzugang in der gRV bezieht eine EM-Rente (DRV 2009b).

\subsection{HÖHERE QUALIFIKATION VERRINGERT DAS FRÜHBEREN- TUNGSRISIKO}

Die Analysen zeigen, dass in jeder Altersgruppe Versicherte mit hoher Qualifikation weitaus seltener als diejenigen mit mittlerer und vor allem diejenigen mit niedriger Qualifikation eine EM-Rente beziehen. Dies gilt sowohl für Frauen als auch insbesondere für Männer. Auffällig ist, dass Männer mit niedriger Qualifikation in den neuen Bundesländern ein besonders hohes Risiko haben, aus gesundheitlichen Gründen eine Erwerbsminderungsrente zu beziehen. Die Qualifikationsunterschiede werden ausgeprägter, wenn nach häufig vorkommenden Diagnosegruppen differenziert wird. Die größten Differenzen in Abhängigkeit von der höchsten schulischen und beruflichen Qualifikation zeigen sich bei den EM-Rentenzugängen wegen Muskel-Skelett-Erkrankungen als auch wegen Herz-Kreislauf-Erkrankungen. Bei den EM-Rentenzugängen aufgrund von psychischen Erkrankungen zeigt sich bei beiden Geschlechtern ein geringerer Qualifikationseffekt. Hieraus lässt sich schlussfolgern, dass durch Arbeitsverdichtung und Stress verursachte psychische Erkrankungen, die insbesondere bei Frauen die häufigste Diagnosegruppe darstellen und, im Unterschied zu den anderen Diagnosegruppen, tendenziell zunehmen, bei Beschäftigten aller Qualifikationsniveaus vorkommen können, mit lediglich geringen Unterschieden zwischen den alten und den neuen Bundesländern. Dieser Befund könnte in Zusammenhang mit der Einführung des EM-Reformgesetzes (Stichwort: Verweisbarkeit auf andere Tätigkeiten) stehen, wonach durch Ver-

Tabelle 4: Risikoquoten (Odds-Ratios) zur Erwerbsminderungsrente nach Diagnosegruppen, Qualifikation und Region (2008) - logistische Regression ${ }^{+}$

\begin{tabular}{|c|c|c|c|c|c|c|}
\hline \multirow[b]{2}{*}{ Erklärende Variablen } & \multicolumn{2}{|c|}{ Herz-Kreislauf-Erkrankungen } & \multicolumn{2}{|c|}{ Muskel-Skelett-Erkrankungen } & \multicolumn{2}{|c|}{ Psychische Erkrankungen } \\
\hline & Männer & Frauen & Männer & Frauen & Männer & Frauen \\
\hline $\begin{array}{l}\text { Niedriges } \\
\text { Qualifikationsniveau }\end{array}$ & $\begin{array}{l}10,03^{*} \\
(0,8472)\end{array}$ & $\begin{array}{c}6,49^{*} \\
(0,9479)\end{array}$ & $\begin{array}{c}14,22^{*} \\
(1,4691)\end{array}$ & $\begin{array}{c}7,93^{*} \\
(0,8049)\end{array}$ & $\begin{array}{c}7,22^{*} \\
(0,3495)\end{array}$ & $\begin{array}{c}3,22^{*} \\
(0,1374)\end{array}$ \\
\hline $\begin{array}{l}\text { Mittleres } \\
\text { Qualifikationsniveau }\end{array}$ & $\begin{array}{c}5,05^{*} \\
(0,4092)\end{array}$ & $\begin{array}{c}3,33^{*} \\
(0,4708)\end{array}$ & $\begin{array}{c}9,72^{*} \\
(0,9715)\end{array}$ & $\begin{array}{c}4,50^{*} \\
(0,4449)\end{array}$ & $\begin{array}{c}3,15^{*} \\
(0,1453)\end{array}$ & $\begin{array}{c}1,88^{*} \\
(0,0757)\end{array}$ \\
\hline Hohes Qualifikationsniveau & \multicolumn{6}{|c|}{ Referenzkategorie } \\
\hline Ostdeutschland & $\begin{array}{c}1,78^{*} \\
(0,0527)\end{array}$ & $\begin{array}{c}1,53^{*} \\
(0,0768)\end{array}$ & $\begin{array}{c}1,68^{*} \\
(0,0462)\end{array}$ & $\begin{array}{c}1,31^{*} \\
(0,0408)\end{array}$ & $\begin{array}{c}1,09 * \\
(0,0262)\end{array}$ & $\begin{array}{c}0,90^{*} \\
(0,019)\end{array}$ \\
\hline Westdeutschland & Ref. & Ref. & Ref. & Ref. & Ref. & Ref. \\
\hline Pseudo- $\mathrm{R}^{2}$ & 0,0954 & 0,0526 & 0,0989 & 0,0756 & 0,0374 & 0,0349 \\
\hline Beobachtungen & 9.504 .952 & 8.339 .746 & 9.504 .952 & 8.339 .746 & 9.504 .952 & 8.339 .746 \\
\hline
\end{tabular}


schärfung der Anspruchsvoraussetzungen ein Zugang in EM-Rente wegen MuskelSkelett-Erkrankungen insbesondere für Männer erschwert wurde. Insgesamt sind zurückgehende EM-Rentenzugänge bei allen Diagnosegruppen zu verzeichnen, mit Ausnahme von psychischen Erkrankungen. Fischer und Irle (2009, S. 150) begründen diese Entwicklung mit verbesserter Diagnostik und Identifizierung psychischer Störungen im Rentenantragsverfahren und gehen insgesamt von einer gestiegenen Akzeptanz von psychischen Störungen aus. Dannenberg et al. (2010, S. 288) behaupten, dass Aufklärung und nachlassende Stigmatisierung dazu beitrügen, „(...) dass Menschen mit psychischen Störungen ihr Leiden z. B. nicht mehr hinter unspezifischen Diagnosen wie „Chronische Rückenschmerzen“ oder anderen somatischen Diagnosen verbergen.“ (Hervorhebung i. O.) Zusammenfassend „schützt“ höhere Qualifikation weniger vor psychischen als vor insbesondere Muskel-Skelett-Erkrankungen, die den stärksten Bildungsgradient aufweisen. Oder umgekehrt: Im Unterschied zu den anderen Diagnosegruppen weisen psychische Erkrankungen vergleichsweise geringe regionale, geschlechts- oder qualifikationsabhängige Spezifika auf.

Die Ursachen gesundheitlicher Ungleichheit sind vielfältig. Zum einen können sie auf gesundheitsbewusstere Lebens- und Verhaltensweisen von Höherqualifizierten zurückgeführt werden (Helmert 2003; Lampert et al. 2005; Nocon et al. 2007), zum anderen können sie in Zusammenhang mit der ausgeübten beruflichen Tätigkeit und den damit verbundenen Risiken stehen. Erwerbstätige mit höherer Qualifikation üben im Vergleich zu Niedrigqualifizierten seltener Berufe aus, in denen chronische körperliche Fehlbelastungen auftreten.

\subsection{MÄNNER WEISEN EIN HÖHERES EM-RISIKO AUF ALS FRAUEN}

Die geschlechterdifferenzierten Analysen bestätigen bisherige Ergebnisse: Männer beziehen durchschnittlich häufiger als Frauen eine EM-Rente. Auffällig ist dabei, dass die Geschlechterdifferenzen in den neuen Bundesländern ausgeprägter sind als in den alten. Hervorzuheben ist außerdem, dass sich das Qualifikationsniveau bei Männern wesentlich stärker auf das Erwerbsminderungsrisiko auswirkt als bei
Frauen. Die Analysen nach Diagnosegruppen ergeben allerdings ein differenzierteres Bild: Während Männer aufgrund von Herz-Kreislauf- sowie von Muskel-SkelettErkrankungen ein erhöhtes EM-Risiko haben, zeigt sich ein umgekehrtes Bild bei den EM-Rentenzugängen aufgrund von psychischen Erkrankungen: hier sind es die Frauen, die ein höheres EM-Risiko im Vergleich zu den Männern haben. Zudem sind die Geschlechterdifferenzen bei psychischen Erkrankungen in den alten Bundesländern höher als in den neuen.

Die Ursachen der geschlechtsspezifischen Unterschiede hinsichtlich des EMRisikos können hier nicht abschließend geklärt werden. Vor dem Hintergrund, dass Berufe mit hohem gesundheitlichen Gefährdungspotenzial mehrheitlich von (niedrig qualifizierten) Männern ausgeübt werden, ist das erhöhte EM-Risiko von Männern aufgrund von Herz-KreislaufErkrankungen und Muskel-Skelett-Erkrankungen plausibel. Das erhöhte Risiko der Frauen, psychisch zu erkranken, könnte damit begründet werden, dass Frauen häufiger als Männer in emotional belastenden Berufen tätig sind, die zu stressbedingten Erkrankungen wie Depressionen und Burnout führen (Beermann et al. 2008; $\mathrm{BiBB} / \mathrm{BAuA} 2006)$. Allerdings ist neben der Risikoexposition auch die unterschiedliche Wahrnehmung und Verarbeitung der psychischen Belastungen von Frauen und Männern zu berücksichtigen. Männer zeigen andere Symptome als Frauen, sie kompensieren ihre Probleme anders und haben ein anderes Hilfesuchverhalten als Frauen (DAK 2008; Möller-Leimkühler/ Kasper 2010).

\subsection{EM-RISIKO IST IN DEN NEUEN BUNDESLÄNDERN HÖHER ALS IN DEN ALTEN}

Hinsichtlich der Differenzen zwischen den alten und neuen Bundesländern fällt auf, dass die regionalen Disparitäten bei Frauen geringer ausfallen als bei Männern. Die Analysen zeigen darüber hinaus, dass insbesondere bei den Frauen die Ost-West-Unterschiede mit zunehmender Qualifikation abnehmen, aber auch bei den hochqualifizierten Männern sind lediglich marginale Ost-West-Unterschiede im Hinblick auf ihr EM-Rentenrisiko festzustellen. Differenziert man die globalen EM-Renten-Risiken nach häufig vorkommenden Diagnosegruppen, zeigt sich bei den Herz-Kreislauf-Erkrankungen sowie den Muskel-Skelett-Erkrankungen ein noch deutlicheres Bild als bei den Zugängen in Erwerbsminderungsrente insgesamt. Sowohl bei Frauen als auch bei Männern ist hier ein stark erhöhtes Risiko eines EM-Rentenzugangs in Ost- im Vergleich zu Westdeutschland zu verzeichnen. Hinsichtlich der EM-Rentenzugänge aufgrund von psychischen Erkrankungen zeigt sich hingegen ein anderes Bild: Hier fallen sowohl die Qualifikations- als auch die OstWest-Unterschiede deutlich geringer aus. Bei Frauen ist das mit psychischen Erkrankungen verbundene EM-Rentenrisiko in den neuen Bundesländern etwas geringer als in den alten Bundesländern. Diese Befunde sind besonders hervorzuheben, weil Erkrankungen aufgrund psychischer Störungen tendenziell und vor allem anteilig zunehmen, während die Bedeutung anderer Diagnosegruppen eher zurückgeht. Psychische Erkrankungen, die vermutlich durch Arbeitsverdichtung, Ängste in Bezug auf die Arbeitsplatzsicherheit oder beruflichen wie privaten Stress verursacht werden, sind sozial weniger selektiv als andere chronische Krankheiten.

Die Unterschiede zwischen den alten und neuen Bundesländern hinsichtlich des EM-Rentenrisikos können nicht abschließend geklärt werden. Das erhöhte Vorkommen der Herz-Kreislauf- und der Muskel-Skelett-Erkrankungen insbesondere bei ostdeutschen Männern könnte darauf zurückzuführen sein, dass überproportional viele Männer in den neuen Bundesländern in Arbeitsverhältnissen mit belastenden Umgebungsfaktoren und physisch belastenden manuellen Tätigkeiten beschäftigt sind oder waren. Zudem ist selektive Binnenmigration, wonach gesündere Ostdeutsche zum Arbeiten in den Westen ziehen, während weniger Gesunde im Osten bleiben, im Sinne des „HealthyMigrant-Effects“ (Abraido-Lanza et al. 1999) vorstellbar.

Im Hinblick auf die Stärke des Einflusses erklärender Variablen ist grundsätzlich festzustellen, dass vor allem die Qualifikation - gefolgt von geschlechtsspezifischen und regionalen Merkmalen - einen wesentlichen Einfluss auf Erwerbsminderungsrisiken hat. Wobei mit zunehmender Qualifikation der Einfluss von Geschlecht und Regionalität deutlich zurückgeht: Hochqualifizierte Männer und Frauen haben vergleichbare EM-Renten-Risiken, die jeweils in den neuen Bundesländern 
geringfügig höher sind. Während bei den geringer Qualifizierten die Männer ein wesentlich höheres EM-Renten-Risiko aufweisen als Frauen.

Die in der vorliegenden Studie präsentierten Ergebnisse zum Zusammenhang von EM-Renten-Risiken und Qualifika- tion liefern auf hinreichenden Fallzahlen basierende valide Befunde zum Themenbereich soziale und gesundheitliche Ungleichheit in Deutschland. Sie verweisen auf die große Bedeutung von schulischer und beruflicher Qualifikation für ein langes und gesundes (Arbeits-)Leben.
Dennoch ist weiterer Forschungsbedarf vorhanden, da sozialmedizinische bzw. epidemiologische Analysen über Frühberentung wegen Krankheit sowie vor allem über mögliche Ursachen des Berentungsgeschehens nach wie vor weitgehend fehlen (Richter 2007).

\section{LITERATUR}

Abraido-Lanza, A. F./Dohrenwend, B. P./Ng-Mak, D. S./Turner, J. B. (1999): The Latino mortality paradox: a test of the "salmon bias" and healthy migrant hypotheses, in: American Journal of Public Health 89 (10), S. 1543-1548

Albrecht, M./Loos, St./Schiffhorst, G. (2007): Sozioökonomische Situation von Personen mit Erwerbsminderung: eine Analyse auf Basis von SOEP-Daten, in: Deutsche Rentenversicherung 62 (10), S. 621-640

Beermann, B./Brenscheidt, F./Siefer, A. (2008): Unterschiede in den Arbeitsbedingungen und -belastungen von Frauen und Männern, in: Badura, B./Schröder, H./Vetter, C. (Hrsg.): Fehlzeiten-Report 2007. Arbeit, Geschlecht und Gesundheit. Zahlen, Daten, Analysen aus allen Branchen der Wirtschaft, S. 67-82

Bundesinstitut für Berufsbildung (BIBB)/Bundesanstalt für Arbeitsschutz und Arbeitsmedizn (BAuA) (2006): Erwerbstätigenbefragung 2005/2006, http://www.bibb.de/de/26901.htm (Zugriff am 09.06.2010)

Brandes, H. (2003): Männlicher Habitus und Gesundheit. Blickpunkt der Mann 1 (2), S. 10-13

Brockmann, H./Müller, R./Helmert, U. (2009): Time to retire - Time to die? A prospective cohort study of the effects of early retirement on long term survival, in: Social Science and Medicine, doi:10.1016/ j.socscimed.2009.04.009

Brussig, M./Wojtkowski, S. (2006): Durchschnittliches Renteneintrittsalter steigt weiter, Altersübergangs-Report 2006-02, hrsg. von: Institut Arbeit und Technik/Forschungsnetzwerk Alterssicherung/Hans-BöcklerStiftung, Gelsenkirchen/Berlin/Düsseldorf

Dannenberg, A./Hofmann, J./Kaldybajewa, K./Kruse, E. (2010): Rentenzugang 2009: Weiterer Anstieg der Zugänge in Erwerbsminderungsrenten wegen psychischer Erkrankungen, in: RVaktuell 9, S. 283-293

Deutsche Angestellten-Krankenkasse (DAK) (2008): Gesundheitsreport 2008. Analyse der Arbeitsunfähigkeitsdaten. Schwerpunktthema Mann und Gesundheit, Hamburg

Deutsche Rentenversicherung (DRV) (2009a): Rentenversicherung in Zeitreihen, Oktober, DRV-Schriften, Band 22

Deutsche Rentenversicherung (DRV) (2009b): Rentenzugang 2008, Statistik der Deutschen Rentenversicherung, Band 173

Dragano, N. (2007): Arbeit, Stress und krankheitsbedingte Frührenten. Zusammenhänge aus theoretischer und empirischer Sicht, Wiesbaden
Dragano, N./Friedel, H./Bödeker, W. (2008): Soziale Ungleichheit bei der krankheitsbedingten Frühberentung, in: Bauer, U./Bittlingmayer, U. H./Richter, M. (Hrsg.): Health Inequalities. Determinanten und Mechanismen gesundheitlicher Ungleichheit, Wiesbaden, S. 108-124 Europäische Agentur für Sicherheit und Gesundheitsschutz am Arbeitsplatz (Hrsg.) (2006): Geschlechtsspezifische Aspekte der Sicherheit und des Gesundheitsschutzes bei der Arbeit. Eine zusammenfassende Darstellung, Amt für amtliche Veröffentlichungen der Europäischen Gemeinschaft, Luxemburg

Fachinger, U./Himmelreicher, R. K. (2007): Alterslohnprofile und Qualifikation in den alten Bundesländern - Eine empirische Analyse auf Datenbasis des Längsschnittdatensatzes SUF VVL 2004, in: Deutsche Rentenversicherung (11-12), S. 750-770

Fischer, K./Irle, H. (2009): Psychische Störungen - Sozialmedizinische Bedeutung und Entwicklung in der medizinischen Rehabilitation, in: RVaktuell 56 (4), S. 149-158

Frommert, D./Himmelreicher R. K. (2010): Angleichung oder zunehmende Ungleichheit? Alterseinkünfte in den alten und den neuen Bundesländern, in: Krause, P./Ostner, I. (Hrsg.): Leben in Ost- und Westdeutschland. Eine sozialwissenschaftliche Bilanz der deutschen Einheit 1990-2010, Frankfurt, M./New York, S. 351-372

Hagen, C./Himmelreicher, R. K./Kemptner, D./Lampert. T. (2010): Soziale Unterschiede beim Zugang in Erwerbsminderungsrente. Eine Analyse auf Datenbasis von Scientific Use Files des Forschungsdatenzentrums der Rentenversicherung, RatSWD, Research Note (44) Helmert, U. (2003): Individuelle Risikofaktoren, Gesundheitsverhalten und Mortalitätsentwicklung in Deutschland im Zeitraum 1984 bis 1998, in: Gesundheitswesen 65, S. 542-547

Himmelreicher, R. K./Stegmann, M. (2008): New possibilities for socio-economic research through longitudinal data from the Research Data Center of the Federal German Pension Insurance (FDZ-RV),

in: Schmollers Jahrbuch 128 (4), S. 647-660

Himmelreicher, R. K./Hagen, C./Clemens, W. (2009): Bildung und Übergang in den Ruhestand: Gehen Höherqualifizierte später in Rente?, in: Kölner Zeitschrift für Soziologie und Sozialpsychologie 61 (3), S. $437-452$

Koalitionsvertrag zwischen CDU, CSU und FDP (2009): Wachstum, Bildung, Zusammenhalt 
Köhler-Rama, T./Lohmann, A./Viebrok, H. (2010): Vorschläge zu einer Leistungsverbesserung bei Erwerbsminderungsrenten aus der gesetzlichen Rentenversicherung, in: Zeitschrift für Sozialreform (ZSR) 56 (1), S. $59-83$

Koppelin, F./Müller, R. (2004): Macht Arbeit Männer krank? Arbeitsbelastungen und arbeitsbedingte Erkrankungen bei Männern und Frauen, in: Altgeld, T. (Hrsg.): Männergesundheit. Neue Herausforderungen für Gesundheitsförderung und Prävention, Weinheim/München, S. $121-134$

Kreikebohm, R./Kuszynski, J. (2011): Der versicherte Personenkreis in der gesetzlichen Rentenversicherung, in: Eichenhofer, E./Rische, H./ Schmähl, W. (Hrsg.): Handbuch der gesetzlichen Rentenversicherung SGB VI, Köln, S. 383-410

Kuhn, K. (2008): Geschlechtsspezifische arbeitsbedingte Gesundheitsgefahren und Erkrankungen, in: Badura, B./Schröder, H./Vetter, C. (Hrsg.):Fehlzeiten-Report 2007. Arbeit, Geschlecht und Gesundheit. Zahlen, Daten, Analysen aus allen Branchen der Wirtschaft, S. 83-96 Kurth, B.-M./Lange, C./Kamtsiuris, P./Hölling, H. (2009): Gesundheitsmonitoring am Robert Koch-Institut. Sachstand und Perspektiven, in: Bundesgesundheitsblatt - Gesundheitsforschung - Gesundheitsschutz 52 (7), S. 557-570

Lademann, J./Kolip, P. (2005): Gesundheit von Frauen und Männern im mittleren Lebensalter, Schwerpunktbericht der Gesundheitsberichterstattung des Bundes, Robert Koch-Institut, Berlin

Lampert, Th./Saß A.-C./Häfelinger, M./Ziese, T. (2005): Armut, soziale Ungleichheit und Gesundheit. Expertise zum 2. Armuts- und Reichtumsbericht der Bundesregierung, Beiträge zur Gesundheitsberichterstattung des Bundes, Robert Koch-Institut, Berlin

Marmot, M./Wilkonson, R. G. (Hrsg.) (1999): Social determinants of health, Oxford

Mielck, A. (2000): Soziale Ungleichheit und Gesundheit. Empirische Ergebnisse, Erklärungsansätze, Interventionsmöglichkeiten, Bern u. a.

Mielck, A. (2005): Soziale Ungleichheit und Gesundheit. Einführung in die aktuelle Diskussion, Bern u.a.
Möller-Leimkühler A. M./Kasper, S. (2010): Psychische und Verhaltensstörungen, in: Bardehle, T./Stiehler, M. (Hrsg.): Erster Deutscher Männergesundheitsbericht. Ein Pilotbericht, München, S. 135-159

Nocon, M./Keil, T./Willich, S. (2007): Education, income, occupational status and health risk behaviour, in: Journal of Public Health 15 (5), S. $401-405$

Radl, J. (2007): Individuelle Determinanten des Renteneintrittsalters. Eine empirische Analyse von Übergängen in den Ruhestand, in: Zeitschrift für Soziologie 36 (1), S. 43-63

Richter, D. (2007): Psychische Störungen und Erwerbsminderungsberentung, in: DRV-Schriften, Band 55, S. 212-223

Richter, M./Hurrelmann, K. (2009): Gesundheitliche Ungleichheit. Grundlagen, Probleme, Perspektiven, Wiesbaden

Robert, S. A./House, J. S. (2000): Socioeconomic inequalities in health: Integrating individual, community-, and societal-level theory and research, in: Albrecht, G. L./Fitzpatrick, R./Scrimshaw, S. C. (Hrsg.): The Handbook of Social Studies in Health and Medicine, London, S. $115-135$

Robert Koch-Institut (RKI) (Hrsg.) (2006): Gesundheit in Deutschland. Gesundheitsberichterstattung des Bundes, Berlin

Robert Koch-Institut (RKI) (Hrsg.) (2009): 20 Jahre nach dem Fall der Mauer: Wie hat sich die Gesundheit in Deutschland entwickelt? Beiträge zur Gesundheitsberichterstattung des Bundes, Berlin Schmidtke, H. (1997): Gesundheitsversorgung und Gesundheit in den neuen Bundesländern, in: Hauser, R./Olk, Th. (Hrsg.): Soziale Sicherheit für alle? Beiträge zu den Berichten zum sozialen und politischen Wandel in Ostdeutschland, Opladen, S. 173-222

Sticher, B. (2010): Riskantes Verhalten, in: Bardehle, T., Stiehler, M. (Hrsg.): Erster Deutscher Männergesundheitsbericht. Ein Pilotbericht, München, S. 87-110

Viebrok, H./Himmelreicher, R. K./Schmähl, W. (2004): Private Altersvorsorge statt Rente: Wer gewinnt, wer verliert?, hrsg. von Schmähl, W.: Beiträge zur Sozial- und Verteilungspolitik, Band 3, Münster u.a. 